\title{
The role of uranium speciation in its isotopic fractionation
}

\author{
RIZLAN BERNIER-LATMANI ${ }^{1, *}$, ASHLEY BROWN ${ }^{1}$, \\ ZEZHEN PAN ${ }^{1}$, RADMILA FAIZOVA ${ }^{2}$, ATARU SATO ${ }^{3}$, \\ YVONNE ROEBBERT ${ }^{4}$, TONYA VITOVA ${ }^{5}$, MARINELLA \\ MAZZANTI $^{2}$, MiNORI ABE ${ }^{3}$, STEFAN WEYER ${ }^{4}$
}

${ }^{1} \mathrm{EML}$ and ${ }^{2} \mathrm{GCC}$, École polytechnique fédérale de Lausanne, Lausanne, 1015, Switzerland ( ${ }^{*}$ rizlan.bernierlatmani@epfl.ch); ${ }^{3}$ Tokyo Metropolitan University, Tokyo, Japan; ${ }^{3}$; ${ }^{4}$ Institut für Mineralogie, Leibniz, Universitat Hannover, D-30167 Hannover, Germany; ${ }^{5}$ Institute for Nuclear Waste Disposal (INE), Karlsruhe Institute of Technology, Karlsruhe 76131, Germany

The uranium (U) biogeochemical cycle includes the oxidation of minerals bearing reduced $U$ (tetravalent $U$, $\mathrm{U}(\mathrm{IV})$ ) by $\mathrm{O}_{2}$ as well as the reduction and immobilization of the resulting hexavalent $\mathrm{U}(\mathrm{U}(\mathrm{VI}))$ in anoxic sediments. The reduction of $\mathrm{U}(\mathrm{VI})$ to $\mathrm{U}(\mathrm{IV})$ is catalyzed by microorganisms as well as by abiotic reducing agents such as reduced minerals and aqueous species. Furthermore, this reduction proceeds via either two one-electron transfers or a oneelectron transfer to pentavalent $\mathrm{U}(\mathrm{U}(\mathrm{V}))$ followed by disproportionation to $\mathrm{U}(\mathrm{VI})$ and $\mathrm{U}(\mathrm{IV})$. Additionally, the isotopic fractionation of $\mathrm{U}$ at equilibrium calls for the preferential reduction of the heavy $\left({ }^{238} \mathrm{U}\right)$ isotope and its accumulation in the reduced phase [1]. Thus, a heavy $U$ signature in sediments is being used to invoke anoxia in paleo and modern environments [2]. Here, we investigate the role of uranium speciation in its isotopic fractionation. We consider a series of systems in which the speciation of both oxidized and reduced $U$ is systematically varied. In those systems, we probe the isotopic fractionation during reduction. We considered the microbial reduction of U(VI) complexed to a synthetic polyaminocarboxylate ligand, DPAEA that stabilizes the U(V) intermediate, or complexed to carbonate in the presence and absence of calcium or to citrate. Furthermore, several U(IV) species were probed. We also considered the abiotic reduction of U(VI) by magnetite. Finally, ligand effects on isotopic fractionation were also investigated using ab initio calculations.

Here, we will present the results of these investigations and evaluate the relationship between speciation and extent of isotopic fractionation as well as identify gaps in the current understanding of uranium isotopic fractionation. The work ultimately aims at better constraining the mechanistic underpinnings of uranium isotopic fractionation.

[1] Bigeleisen, J. Am. Chem. Soc. 118, 3676-3680, 1996

[2] Montoya-Pino, Geology, 38, 315-318, 2010 